\title{
Particle beam eigenemittances, phase integral, vorticity, and rotations
}

\author{
L. Groening $\odot$ and C. Xiao* \\ GSI Helmholtzzentrum für Schwerionenforschung GmbH, Darmstadt D-64291, Germany \\ M. Chung $\mathbb{( 1 0}^{\dagger}$ \\ Ulsan National Institute of Science and Technology, Ulsan 44919, Republic of Korea
}

(Received 24 December 2020; accepted 7 May 2021; published 24 May 2021)

\begin{abstract}
Particle beam eigenemittances comprise the lowest set of rms-emittances that can be imposed to a beam through symplectic optical elements. For cases of practical relevance this paper introduces an approximation providing a very simple and powerful relation between transverse eigenemittance variation and the beam phase integral. This relation enormously facilitates modeling eigenemittance tailoring scenarios. It reveals that difference of eigenemittances is given by the beam phase integral or vorticity rather than by angular momentum. Within the approximation any beam is equivalent to two objects rotating at angular velocities $\pm \omega$. A description through circular beam modes has been done already in [A. Burov, S. Nagaitsev, and Y. Derbenev, Circular modes, beam adapters, and their applications in beam optics, Phys. Rev. E 66, 016503 (2002)]. The new relation presented here is a complementary and vivid approach to provide a physical picture of the nature of eigenemittances for cases of practical interest.
\end{abstract}

DOI: 10.1103/PhysRevAccelBeams.24.054201

\section{INTRODUCTION}

The terms of eigenemittances have been introduced by A.J. Dragt in 1992 [1] as the projected rms-emittances a beam acquires after all correlations between the degrees of freedom (planes) have been removed. Accordingly, they form the set of lowest projected beam emittances which can be achieved by applying symplectic and linear beam line elements. Since this set may not fit requirements of certain beam applications, tailoring of eigenemittances became a subject of extensive theoretical and experimental research. The first proposal of eigenemittance modification was made in [2] followed by other fundamental investigations [3-15] and experimental applications in linear electron [16-19] and ion accelerators [20-22]. Measurements of eigenemittances and beam coupling have been reported in [23-28] for instance.

Albeit the underlying theory is well understood and experimental results match analytical calculations and layouts, the physical nature of eigenemittances lacks a picture being more vivid compared to the common sense definition of "projected rms-emittances after removal of interplane correlations". For some special cases simple relations were derived as for beams with cylindrical symmetry dominated

\footnotetext{
*la.groening@gsi.de

mchung@unist.ac.kr
}

Published by the American Physical Society under the terms of the Creative Commons Attribution 4.0 International license. Further distribution of this work must maintain attribution to the author(s) and the published article's title, journal citation, and DOI. by their angular momentum, for which the difference of the two eigenemittance is equal to the angular momentum and their mean is the transverse rms-emittance [4].

This general lack of tangible comprehension is partially due to the fact that already the two transverse eigenemittances are calculated in the four-dimensional (4d) phase space spanned by two space coordinates and two momentum coordinates, not to mention the three eigenemittances of a beam including also longitudinal coordinates. This paper intends to shrink this lack of vivid understanding at least for the two transverse eigenemittances.

In the next section basic terms are defined and the derivation of the extended Busch theorem is revised and adapted for further use. The third section derives the role of beam vorticity and the phase integral for transverse eigenemittances. Section IV introduces an approximation that is valid for cases of practical interest. It shows that in general it is vorticity being relevant for the difference of eigenemittances rather than angular momentum. Afterwards several applications demonstrate the practical power of the beam phase integral on eigenemittance calculation. Section VI shows that eigenemittances can be taken practically as equivalents of two areas rotating with same angular velocity but with opposite signs. The paper closes with a conclusion and an outlook.

\section{BASIC TERMS AND EXTENDED BUSCH THEOREM}

The two transverse eigenemittances $\varepsilon_{1 / 2}$ originally introduced in [1] are equal to the two projected transverse beam 
rms-emittances $\varepsilon_{x / y}$, if and only if there are no correlations between the two transverse planes. Eigenemittances can be obtained by solving the complex equation

$$
\operatorname{det}\left(J C-i \varepsilon_{1 / 2} I\right)=0,
$$

where $I$ is the identity matrix,

$$
C=\left[\begin{array}{cccc}
\left\langle x^{2}\right\rangle & \left\langle x x^{\prime}\right\rangle & \langle x y\rangle & \left\langle x y^{\prime}\right\rangle \\
\left\langle x x^{\prime}\right\rangle & \left\langle x^{\prime 2}\right\rangle & \left\langle y x^{\prime}\right\rangle & \left\langle x^{\prime} y^{\prime}\right\rangle \\
\langle x y\rangle & \left\langle y x^{\prime}\right\rangle & \left\langle y^{2}\right\rangle & \left\langle y y^{\prime}\right\rangle \\
\left\langle x y^{\prime}\right\rangle & \left\langle x^{\prime} y^{\prime}\right\rangle & \left\langle y y^{\prime}\right\rangle & \left\langle y^{\prime 2}\right\rangle
\end{array}\right],
$$

and

$$
J=\left[\begin{array}{cccc}
0 & 1 & 0 & 0 \\
-1 & 0 & 0 & 0 \\
0 & 0 & 0 & 1 \\
0 & 0 & -1 & 0
\end{array}\right]
$$

where $(x, y)$ denote the particle position coordinates and $\left(x^{\prime}, y^{\prime}\right)$ their derivatives $\left(x^{\prime}, y^{\prime}\right)$ with respect to the longitudinal direction $\vec{s}$. Second moments $\langle u v\rangle$ are defined through a normalized distribution function $f_{b}$ as

$\langle u v\rangle=\iiint \int f_{b}\left(x, x^{\prime}, y, y^{\prime}\right) \cdot u v \cdot d x d x^{\prime} d y d y^{\prime}$.

The two transverse eigenemittances can be calculated as [13]

$$
\varepsilon_{1 / 2}=\frac{1}{2} \sqrt{-\operatorname{tr}\left[(C J)^{2}\right] \pm \sqrt{\operatorname{tr}^{2}\left[(C J)^{2}\right]-16 \varepsilon_{4 d}^{2}}}
$$

being equivalent to [29]

$$
\varepsilon_{1 / 2}=\sqrt{-\frac{\operatorname{tr}\left[(C J)^{2}\right]}{8}+\frac{\varepsilon_{4 d}}{2}} \pm \sqrt{-\frac{\operatorname{tr}\left[(C J)^{2}\right]}{8}-\frac{\varepsilon_{4 d}}{2}}
$$

with $\varepsilon_{4 d}^{2}:=\operatorname{det} C$. Projected transverse beam rms-emittances are defined as [30]

$$
\begin{aligned}
& \varepsilon_{x}^{2}=\left\langle x^{2}\right\rangle\left\langle x^{\prime 2}\right\rangle-\left\langle x x^{\prime}\right\rangle^{2}, \\
& \varepsilon_{y}^{2}=\left\langle y^{2}\right\rangle\left\langle y^{\prime 2}\right\rangle-\left\langle y y^{\prime}\right\rangle^{2} .
\end{aligned}
$$

Busch's original theorem is from stating preservation of longitudinal single particle angular momentum in a magnetic field region with cylindrical symmetry using conjugated momentum [31]. The extended theorem is derived from restating preservation of many-particle beam eigenemittances under symplectic transformations using conjugated momenta [32]. It reads

$$
\begin{aligned}
& \left(\varepsilon_{1}-\varepsilon_{2}\right)^{2}+\left[\frac{A B_{s}}{(B \rho)}\right]^{2} \\
& +2 \frac{B_{s}}{(B \rho)}\left[\left\langle y^{2}\right\rangle\left\langle x y^{\prime}\right\rangle-\left\langle x^{2}\right\rangle\left\langle y x^{\prime}\right\rangle+\langle x y\rangle\left(\left\langle x x^{\prime}\right\rangle-\left\langle y y^{\prime}\right\rangle\right)\right] \\
& \quad=\text { const }
\end{aligned}
$$

where

$$
A:=\sqrt{\left\langle x^{2}\right\rangle\left\langle y^{2}\right\rangle-\langle x y\rangle^{2}}
$$

is the rms-area of the beam (Fig. 1), $B_{s}$ is the longitudinal magnetic field along the beam axis, and $(B \rho)$ is the beam magnetic rigidity. The first term of Eq. (9) is the squared difference of eigenemittances and the second term is the square of the magnetic flux through the beam rms-area $\vec{A}$. Within the subsequent section the meaning of the essential part of the last term

$\mathcal{W} A:=\left\langle y^{2}\right\rangle\left\langle x y^{\prime}\right\rangle-\left\langle x^{2}\right\rangle\left\langle y x^{\prime}\right\rangle+\langle x y\rangle\left(\left\langle x x^{\prime}\right\rangle-\left\langle y y^{\prime}\right\rangle\right)$

is rederived by adapting the method reported in [32] to further use.

\section{BEAM VORTICITY AND PHASE INTEGRAL}

This section starts with proving the relation

$$
\mathcal{W}=\int_{A}\left[\vec{\nabla} \times \overrightarrow{r^{\prime}}(x, y, s)\right] \cdot d \vec{A},
$$

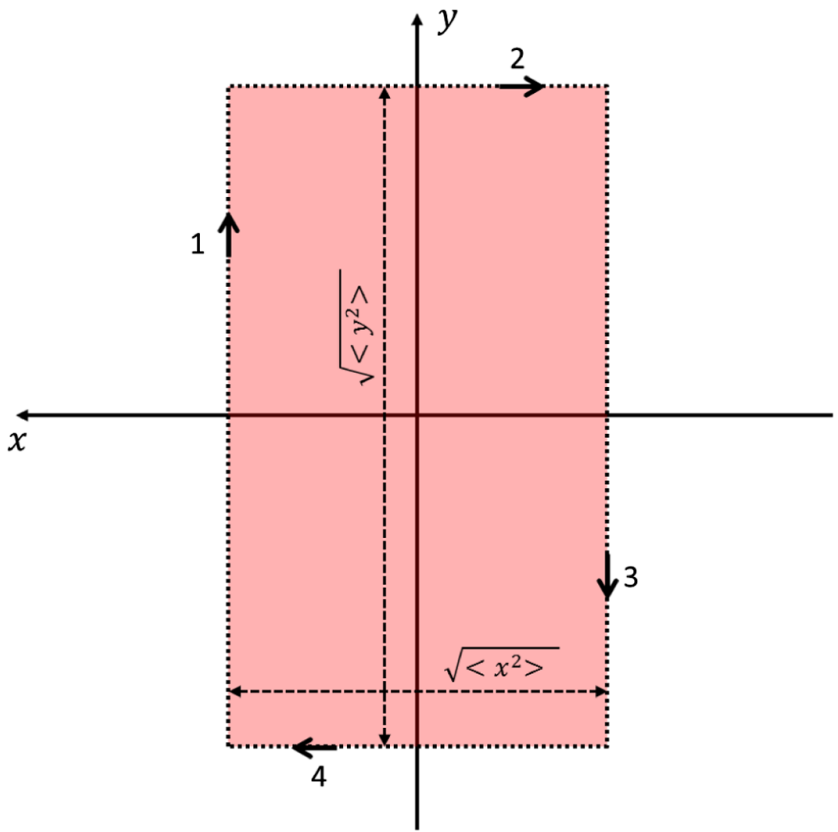

FIG. 1. Definition of the beam rms-area for the case of $\langle x y\rangle=0$ and of the four path sections $P_{i}$ comprising the integral enclosing this area. 
where $\overrightarrow{r^{\prime}}(x, y, s):=\left[\overline{x^{\prime}}(x, y, s), \overline{y^{\prime}}(x, y, s), 1\right]$. This relation states that the third term of Eq. (9) is mainly the mean rmsvorticity $\left(\vec{\nabla} \times \overrightarrow{\overrightarrow{r^{\prime}}}\right)$ integrated over the beam rms-area.

By construction, $\mathcal{W}$ from Eq. (12) is invariant under rotation. Hence, Eq. (12) can be expanded for $\langle x y\rangle=0$ without loss of generality (by assuming that prior to determination of $\mathcal{W}$ the beam is rotated around the beam axis by an angle that puts $\langle x y\rangle$ to zero [33]). Figure 1 illustrates this beam rms-area and the phase integral enclosing it. The transverse components of $\overrightarrow{r^{\prime}}$ are expressed through

$$
\begin{aligned}
& \overline{x^{\prime}}(x, y):=\frac{\left\langle x^{\prime} x\right\rangle}{\left\langle x^{2}\right\rangle} x+\frac{\left\langle x^{\prime} y\right\rangle}{\left\langle y^{2}\right\rangle} y, \\
& \overline{y^{\prime}}(x, y):=\frac{\left\langle y^{\prime} x\right\rangle}{\left\langle x^{2}\right\rangle} x+\frac{\left\langle y^{\prime} y\right\rangle}{\left\langle y^{2}\right\rangle} y .
\end{aligned}
$$

Figure 2 illustrates as an example the constant slope $\partial \overline{x^{\prime}} / \partial y$ of $\overline{x^{\prime}}$ in the projection of the four-dimensional rms-ellipsoid onto the $\left(y, x^{\prime}\right)$ plane. Accordingly,

$$
\vec{\nabla} \times \overrightarrow{r^{\prime}}=\frac{\left\langle y^{\prime} x\right\rangle}{\left\langle x^{2}\right\rangle}-\frac{\left\langle y x^{\prime}\right\rangle}{\left\langle y^{2}\right\rangle}
$$

not explicitly depending on $(x, y)$ hence turning the integral into simple multiplication with $A$ (using $\langle x y\rangle=0$ ) and to

$$
\begin{aligned}
A \mathcal{W} & =A^{2}\left(\vec{\nabla} \times \overrightarrow{r^{\prime}}\right) \\
& =\left\langle x^{2}\right\rangle\left\langle y^{2}\right\rangle\left(\vec{\nabla} \times \overrightarrow{\vec{r}^{\prime}}\right)=\left\langle y^{2}\right\rangle\left\langle y^{\prime} x\right\rangle-\left\langle x^{2}\right\rangle\left\langle y x^{\prime}\right\rangle,
\end{aligned}
$$

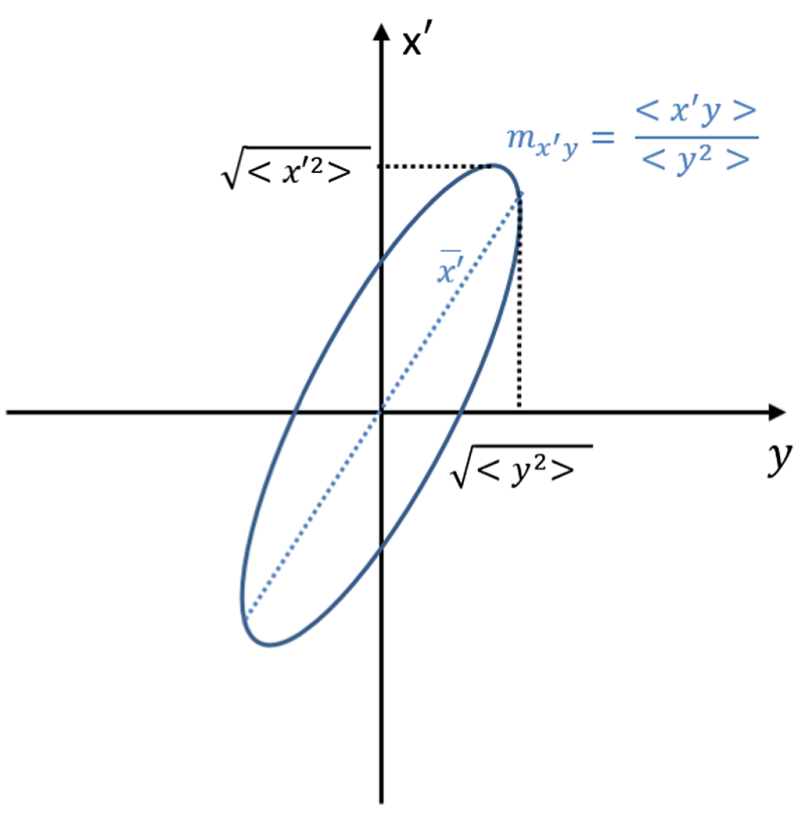

FIG. 2. Projection of the four-dimensional rms-ellipsoid onto the $\left(y, x^{\prime}\right)$ plane and the constant slope $\partial \overline{x^{\prime}} / \partial y$. which had to be proven. Using Stokes' theorem the quantity $\mathcal{W}$ is introduced as

$$
\mathcal{W}=\int_{A}\left[\vec{\nabla} \times \overrightarrow{r^{\prime}}\right] \cdot d \vec{A}=\oint_{\mathcal{C}} \overrightarrow{\vec{r}^{\prime}} \cdot d \vec{C}
$$

being the beam phase integral. The r.h.s. of the equation refers to the integral enclosing the beam rms-area.

Equation (9) is accordingly rephrased as

$$
(\Delta \varepsilon)^{2}+2 \frac{A B_{s}}{(B \rho)} \mathcal{W}+\left[\frac{A B_{s}}{(B \rho)}\right]^{2}=\text { const, }
$$

with $\Delta \varepsilon:=\left(\varepsilon_{1}-\varepsilon_{2}\right)$, being the extended Busch theorem reformulated using the beam phase integral. For constant beam rigidity this expression is equivalent to

$$
\begin{aligned}
& \delta\left((\Delta \varepsilon)^{2}\right)+2 \frac{\mathcal{W}}{(B \rho)}\left(A \delta B_{s}+B_{s} \delta A\right) \\
& \quad+2 \frac{A B_{s}}{(B \rho)} \delta W+2 \frac{A B_{s}}{(B \rho)^{2}}\left(A \delta B_{s}+B_{s} \delta A\right)=0
\end{aligned}
$$

\section{APPROXIMATIONS IN REAL APPLICATIONS}

The extended Busch theorem as stated above is exact. This section illustrates that the actual circumstances of experimental scenarios allow to derive powerful and simple relations from the theorem.

Experimental applications at beam lines with limited apertures keep as short as possible the sections along which eigenemittances shall be modified. This is from the fact that these modifications require imposed correlations, which blow up projected emittances and beam sizes. Accordingly, the beam rms-area along such short sections can be approximated as constant $(\delta A=0)$. Second, the assumption is made that for the above motivated practicality, behind such sections the beam is again fully uncorrelated, i.e., $\mathcal{W}$ vanishes according to Eq. (11). Together with the above prerequisites the assumption is made that change of vorticity is strongly dominated and basically given by the change of the longitudinal magnetic field

$$
-\delta \mathcal{W}:=A \frac{\delta B_{s}}{(B \rho)}
$$

being equivalent to the statement

$$
\frac{A B_{s}}{(B \rho)}+\mathcal{W}:=\text { const. }
$$

Plugging this statement into above Eq. (19) delivers $\delta\left((\Delta \varepsilon)^{2}\right)=\delta\left(\mathcal{W}^{2}\right)$ and accordingly the simple and useful relation 


$$
(\Delta \varepsilon)^{2}-\mathcal{W}^{2}=\text { const. }
$$

The constant is given by the values being evaluated from the second beam moments at any location along the beam line defined as "initial". For practical reasons this will be usually at a position where the beam is uncorrelated, i.e., $(\Delta \varepsilon)_{\text {ini }}$ being equal to the difference of rms-emittances, $\mathcal{W}=0$ and hence const $=(\Delta \varepsilon)_{\mathrm{ini}}^{2}$. The above relation states that the change in the difference of eigenemittances (squared) is equal to the change of integrated beam vorticity (squared). It is in line with the fact that short tilted quadrupoles and dipoles, which change the angular momentum but not the vorticity, do not change the eigenemittances either. But solenoid fringe fields impose beam rotation, i.e., vorticity and do change the beam eigenemittances. The difference between angular momentum and vorticity will be further addressed throughout the subsequent sections and the Appendix.

Throughout this paper the validity of Eqs. (21) and (22) is assumed under the prerequisites stated above, i.e., constant beam rms-area and complete decoupling shortly after tailoring of eigenemittances has been accomplished. The relevance of the latter assumption becomes clear from the formulation of $\mathcal{W}$ as phase integral: if there remains some coupling and the beam passes a long drift, $\mathcal{W}$ might change since $\overrightarrow{r^{\prime}}$ remains constant along the drift but the rmsarea $A$ varies in shape and size. Eigenemittances in turn do not change along any drift. Accordingly, the assumption of full decoupling after intended eigenemittance modification is essential for the application of Eqs. (21) and (22) after the decoupling. However, the intended tailoring process itself is described correctly by the relations.

In the following the contents of Eqs. (21) and (22) are benchmarked by applying them to several scenarios. They are proofed to full generality for cylindrical symmetric beams in solenoids dominated by their angular momentum.

\section{APPLICATIONS}

This section performs applications and hence testing of the relations stated above. First, the relations' properties with respect to symplecticity are investigated followed by calculation of eigenemittances of simple objects, the exact description of cylindrical symmetric beams, a simulation of a nonsymmetric beam scenario, and successful modeling of experiments on eigenemittance tailoring performed at FERMILAB and at GSI.

\section{A. Symplecticity}

The first check of Eq. (22) is on verification whether symplectic transformations leave invariant the vorticity as they leave invariant eigenemittances. Assuming a given transport matrix $M$ the absolute amount of vorticity will change through $M$ by
$\left|A \delta\left(\vec{\nabla} \times \overrightarrow{r^{\prime}}\right)\right|=A\left|\frac{d}{d x} \delta y^{\prime}-\frac{d}{d y} \delta x^{\prime}\right|=A\left|m_{41}-m_{23}\right|$.

The property $m_{41}-m_{23}=0$ is fulfilled by commonly used optical elements being linear and symplectic in the full $4 d$ transverse phase space, as drifts, dipoles, quadrupoles, solenoids, rf-gaps, and even by (nonsymplectic) homogeneous central field regions inside of a solenoid [34], as they feature $m_{41}=m_{23}=0$.

Instead, fringe fields of solenoids are not symplectic, i.e., $m_{41}=-m_{23} \neq 0$. They trigger additional beam vorticity of

$$
\left|A \delta\left(\vec{\nabla} \times \overrightarrow{r^{\prime}}\right)\right|=A\left|m_{41}-m_{23}\right|=A \frac{\left|\delta B_{s}\right|}{(B \rho)},
$$

where $\delta B_{s}$ is the change of field strength along the fringe region. Accordingly, and again in full agreement to Eq. (22), solenoid fringe fields change the eigenemittances as they change the vorticity. They lower one eigenemittance and increase the other one (see Fig. 2 of [13] for instance). In fact the change of difference of eigenemittances $\delta\left((\Delta \epsilon)^{2}\right)$ imposed by a solenoid fringe field corresponding to a longitudinal field variation of $\delta B_{s}$ is according to Eqs. (20) and (22)

$$
\delta\left[(\Delta \epsilon)^{2}\right]=A^{2} \delta\left[\frac{B_{s}}{(B \rho)}\right]^{2} .
$$

The same expression is obtained by doing the lengthy derivation using Eqs. (2), (3), (5) stated at the beginning of Sec. II together with the matrix of a solenoid fringe field [13].

Equation (23) can be very conveniently applied to short beam line elements along which the beam rms-area $A$ is approximated as constant. This is the case for solenoid fringe fields as well as for tapered foils for instance. The latter were proposed for tailoring eigenemittances by inducing horizontal to longitudinal coupling [12]. For short elements the change of difference of eigenemittances simplifies to

$$
\delta\left[(\Delta \varepsilon)^{2}\right]=A^{2}\left(m_{41}-m_{23}\right)^{2} .
$$

Applied to the tapered foil scenario the equation reads

$$
\delta\left[(\Delta \varepsilon)^{2}\right]=A_{x s}^{2}\left(m_{61}-m_{25}\right)^{2},
$$

with $A_{x s}$ as the horizontal-longitudinal pendant of $A$ defined in Eq. (10). The tapered foil causes vorticity in the $(\mathrm{s}, \mathrm{x})$-plane as a solenoid fringe field does in the $(\mathrm{x}, \mathrm{y})$-plane.

\section{B. Rigidly rotating object}

Equation (22) is used to calculate the eigenemittance of a rigidly rotating two dimensional object depicted in Fig. 3. 


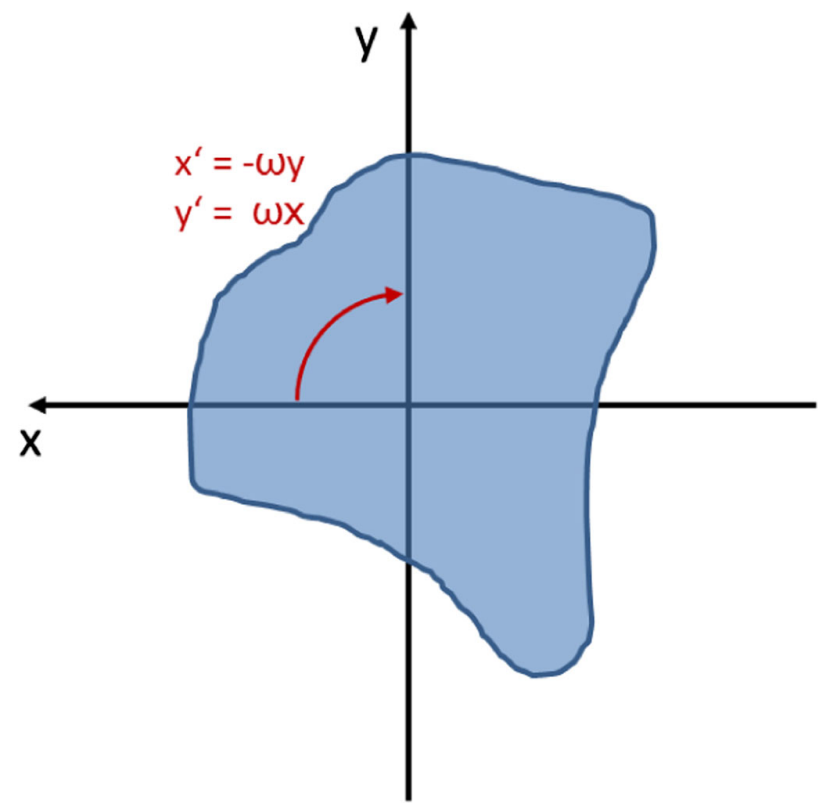

FIG. 3. Arbitrary object rotating rigidly with a constant angular velocity $\omega$ around the beam axis.

On this object the particle coordinates are $(x,-\omega y, y, \omega x)$, where the unit of $\omega$ is $1 / m$ since $s$ is varied along the beam line. Prior to rotation the object was at rest and both eigenemittances as well as its vorticity were equal to zero. Rotation by $\omega$ causes the vorticity $2 \omega$ and in order to satisfy Eq. (22) it causes a difference of eigenemittances of $2|\omega| A$ with $A$ as rms-area [see Eq. (10)]. Applying Eqs. (2), (3), (5) reveals $\varepsilon_{1}=2|\omega| A$ and $\varepsilon_{2}=0$ confirming the above result.

\section{Shearing object}

In an analogue way the eigenemittances of a shearing object depicted in Fig. 4 are calculated. The coordinates are $(x, a y, y, 0)$ which by applying Eq. (22) results in the eigenemittance difference of $|a| A$. The same result is obtained by using Eqs. (2), (3), (5), namely $\varepsilon_{1}=|a| A$ and $\varepsilon_{2}=0$.

\section{Beams with cylindrical symmetry}

Beams with cylindrical symmetry feature $\langle x y\rangle=0$, $\left\langle x^{2}\right\rangle=\left\langle y^{2}\right\rangle=A$, and $\epsilon_{r m s}:=\epsilon_{x}=\epsilon_{y}$. Kim [4] has shown that in this case

$$
\epsilon_{1 / 2}=\epsilon_{r m s} \pm \mathcal{L}
$$

with $2 \mathcal{L}:=\left\langle x y^{\prime}\right\rangle-\left\langle x^{\prime} y\right\rangle$ and accordingly

$$
\epsilon_{1}-\epsilon_{2}=\Delta \epsilon=2 \mathcal{L} \text {. }
$$

Using Eqs. (11) and (12) delivers

$$
\left\langle y^{2}\right\rangle\left\langle x y^{\prime}\right\rangle-\left\langle x^{2}\right\rangle\left\langle x^{\prime} y\right\rangle=A \int_{A}\left[\vec{\nabla} \times \overrightarrow{r^{\prime}}\right] \cdot d \vec{A},
$$

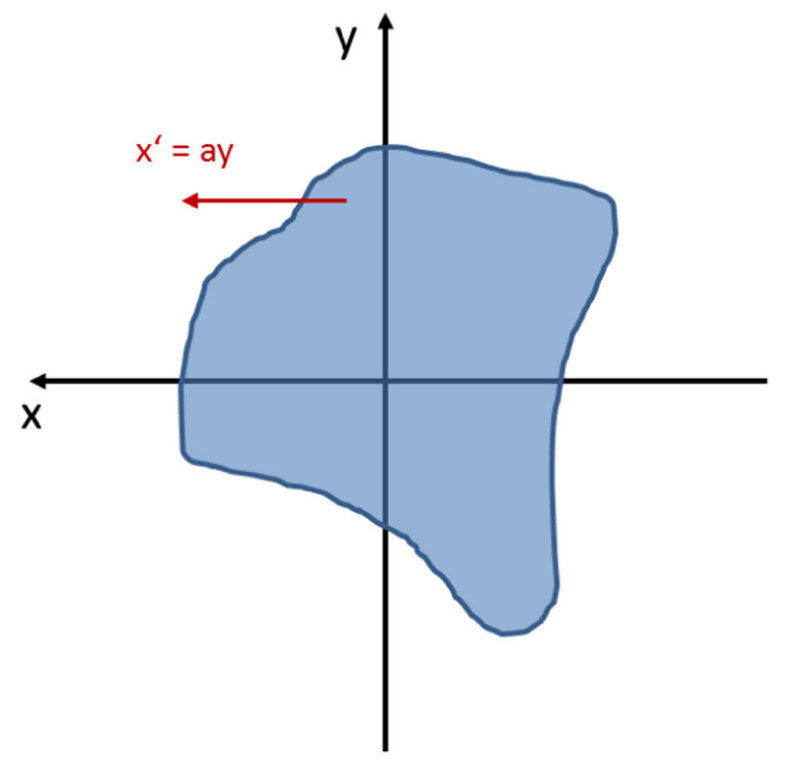

FIG. 4. Arbitrary area at a constant horizontal shear with strength $a$.

which by exploiting the cylindrical symmetry properties stated above together with Eq. (29) gives

$$
\Delta \epsilon=2 \mathcal{L}=\left\langle x y^{\prime}\right\rangle-\left\langle y x^{\prime}\right\rangle=\int_{A}\left[\vec{\nabla} \times \overrightarrow{r^{\prime}}\right] \cdot d \vec{A},
$$

which after taking the square of both sides corresponds to Eq. (22) with the constant being equal to zero. This had to be proven for cylindrical symmetric beams.

Equation (28) is a special case of Eq. (22) with const $=0$ and $\mathcal{W}=2 \mathcal{L}$. In fact, for round objects rotating with $\omega=\omega(r)$ the relation $\mathcal{W}=2 \mathcal{L}$ applies, which is not the case for nonround objects. Accordingly, Eq. (22) is a more general form of Eq. (28), with the former being valid also for beams without cylindrical symmetry and the role of angular momentum $2 \mathcal{L}$ is taken over by the beam phase integral $\mathcal{W}$.

\section{E. Tracking of a nonsymmetric coupled beam}

Finally an initially uncoupled beam has been rmstracked using linear transport matrices through a beam line comprising coupling elements such as solenoids and skewed quadrupoles. Initial beam parameters are listed in Table I and the beam line elements are listed in Table II.

Figures 5 to 7 depict several beam parameters as functions of the position along the beam line. Rmsenvelopes are shown in Fig. 5. They are hardly affected by the solenoids. Figure 6 compares the difference of eigenemittances and the beam phase integral. Both vary just along regions with nonvanishing longitudinal magnetic field. The difference of their squares remains practically constant along the complete beam line. This behavior is in agreement with Eq. (22). 
TABLE I. Initial beam parameters of the tracking calculations.

\begin{tabular}{lc}
\hline \hline Parameter & Value \\
\hline Kinetic energy & $11.45 \mathrm{MeV} / \mathrm{u}$ \\
Mass number & 14 \\
Charge number & 4 \\
$\varepsilon_{x}$ & $4.0 \mathrm{~mm} \mathrm{mrad}$ \\
$\varepsilon_{y}$ & $2.0 \mathrm{~mm} \mathrm{mrad}$ \\
$\beta_{x}$ & $3.0 \mathrm{~m}$ \\
$\alpha_{x}$ & 1.5 \\
$\beta_{y}$ & $1.0 \mathrm{~m}$ \\
$\alpha_{y}$ & -2.0 \\
\hline \hline
\end{tabular}

TABLE II. Beam line of the tracking calculations: element type, length, longitudinal magnetic field, magnet field gradient, and rotation angle around the positive beam axis.

\begin{tabular}{|c|c|c|c|c|}
\hline Element & $\mathrm{L}[\mathrm{m}]$ & $\mathrm{B}[\mathrm{T}]$ & $\mathrm{B}^{\prime}[\mathrm{T} / \mathrm{m}]$ & $\begin{array}{c}\text { Rotation } \\
\text { angle [deg] }\end{array}$ \\
\hline Drift & 0.3 & 0 & 0 & 0 \\
\hline Solenoid & 0.2 & 1.0 & 0 & 0 \\
\hline Drift & 0.3 & 0 & 0 & 0 \\
\hline $\begin{array}{l}\text { Horizontally focusing } \\
\text { quadrupole }\end{array}$ & 0.1 & 0 & 25.0 & 90 \\
\hline Drift & 0.05 & 0 & 0 & 0 \\
\hline $\begin{array}{l}\text { Horizontally focusing } \\
\text { quadrupole }\end{array}$ & 0.1 & 0 & 20.0 & 0 \\
\hline Drift & 0.05 & 0 & 0 & 0 \\
\hline $\begin{array}{l}\text { Horizontally focusing } \\
\text { quadrupole }\end{array}$ & 0.1 & 0 & 20.0 & 45 \\
\hline Drift & 0.05 & 0 & 0 & 0 \\
\hline $\begin{array}{l}\text { Horizontally focusing } \\
\text { quadrupole }\end{array}$ & 0.1 & 0 & 20.0 & -45 \\
\hline Drift & 0.3 & 0 & 0 & 0 \\
\hline Solenoid & 0.55 & 0.5 & 0 & 0 \\
\hline Drift & 0.3 & 0 & 0 & 0 \\
\hline
\end{tabular}

Figure 7 plots the eigenemittances, negative phase integral $-\mathcal{W},\left(A B_{s}\right) /(B \rho)$, and angular momentum $L=$ $\left\langle x y^{\prime}\right\rangle-\left\langle y x^{\prime}\right\rangle=2 \mathcal{L}$ along the beam line. Up to the exit of the first solenoid $-\mathcal{W}$ and $\left(A B_{s}\right) /(B \rho)$ are equal to each other as stated by Eq. (21). Afterwards there is slight augmentation of $-\mathcal{W}$ starting behind the first solenoid. This augmentation is from the finite length of the solenoid for which its exit fringe field does not completely remove vorticity and coupling imposed by the entrance fringe field and thus the beam is coupled behind the solenoid. Along the subsequent beam line this coupling in turn varies the phase integral $\mathcal{W}$ as described in Sec. IV. It must be stressed that this increase of vorticity occurs after the eigenemittance tailoring itself has been accomplished inside the solenoid. Real scenarios sufficiently remove coupling and hence vorticity immediately after having tailored the eigenemittances. For these reasons the mentioned increase shown in Fig. 7 has no impact on quantitative modeling of

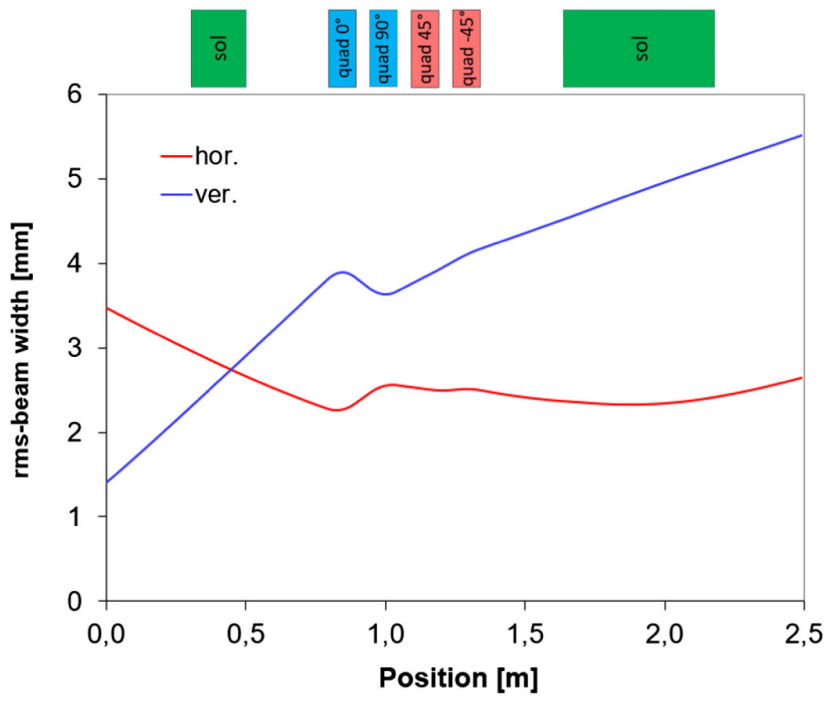

FIG. 5. Horizontal (red) and vertical (blue) rms-envelope along the beam line as obtained from tracking calculations.

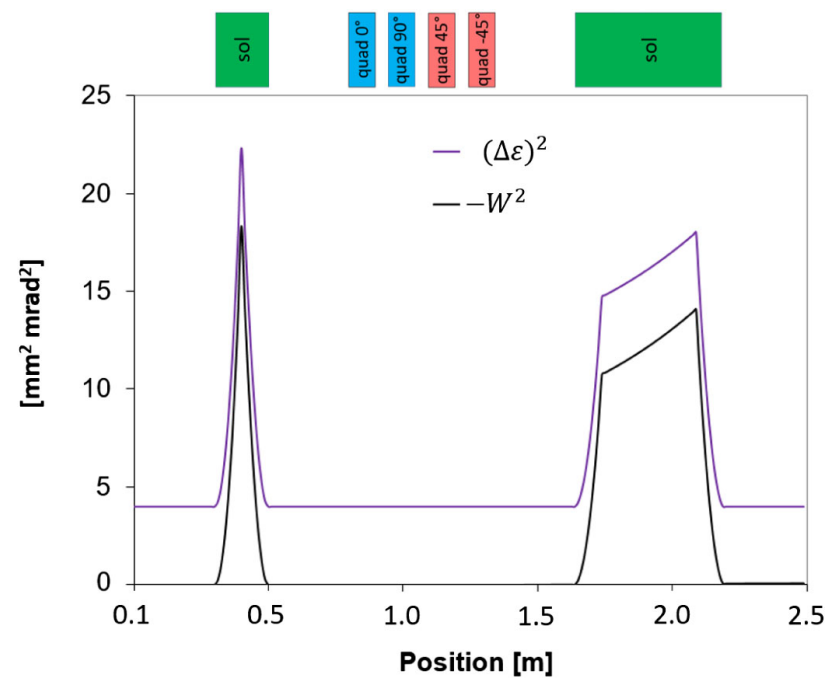

FIG. 6. Squared difference of eigenemittances (violet) and squared beam phase integral (black) as obtained from tracking calculations.

the tailoring, which therefore is done correctly. The tailoring is made through the fringe regions and not through the main field region. Accordingly, real scenarios keep the solenoid main region as short as possible.

Figure 7 also emphasizes the difference between beam path integral $\mathcal{W}$ and angular momentum $L$. Both are equal to each other up to the first quadrupole. Afterwards these two quantities differ significantly and the absolute value of angular momentum exceeds the one of the beam phase integral. The difference of eigenemittances is given by the beam path integral rather than by the angular momentum. This is an important statement of Eq. (22) and an essential difference to Eq. (28). The latter assigns this role to angular momentum and applies just to beams with cylindrical 


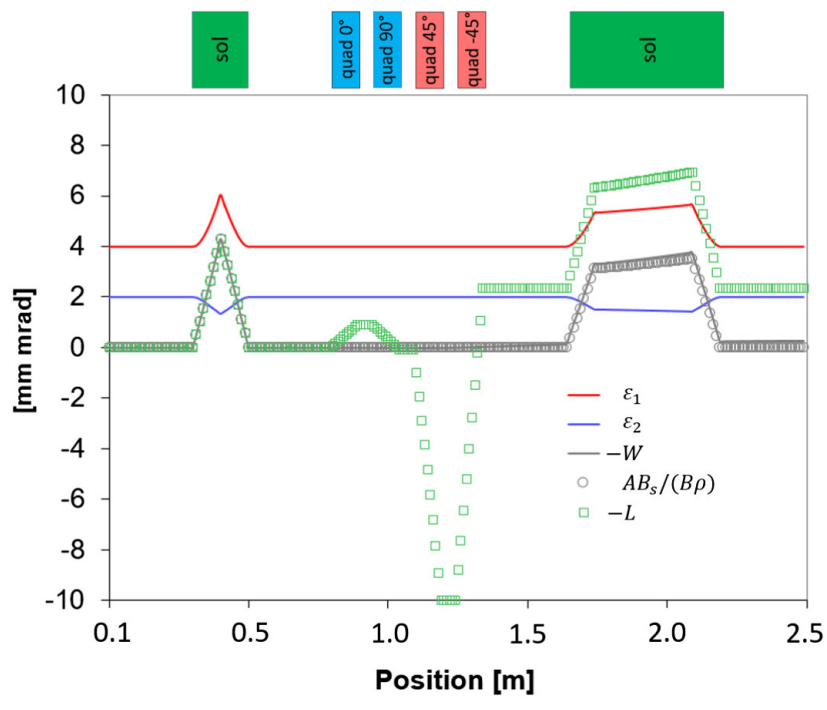

FIG. 7. Eigenemittances (red and blue lines), negative beam phase integral (gray line), $A B_{s} /(B \rho)$ (gray circles) and angular momentum (green boxes) as obtained from tracking calculations.

symmetry. The Appendix gives two illustrative examples on the difference of angular momentum and beam phase integral and their role on eigenemittances.

Finally, it is noted that albeit $\delta A \approx 0$ is not fulfilled along the complete line, Fig. 6 demonstrates that Eq. (22) holds very well. The next two subsections are on modeling of eigenemittance variation performed in experiments.

\section{F. Flat beam experiment at FERMILAB}

At FERMILAB's NICCAD photoinjector an electron beam has been created on a cathode surface of area $A_{0}$ being immersed into a longitudinal magnetic field $B_{0}$ [18]. The beam has been extracted into a region without magnetic field, accelerated, and transversely decoupled. Applying Eqs. (21) and (22), the final difference of normalized eigenemittances can be calculated very quickly.

Since the beam was born with $\Delta \varepsilon_{i}=0$ and $\mathcal{W}_{i}=0$, the constant of Eq. (22) is equal to zero. Hence applying this equation to the beam before and after extraction from the cathode magnetic field region gives

$$
0-0=\left(\Delta \varepsilon_{f}\right)^{2}-\mathcal{W}_{f}^{2},
$$

i.e., $\Delta \varepsilon_{f}=\mathcal{W}_{f}$. This is used in Eq. (21) being also applied to the beam before and after extraction delivering

$$
\frac{A_{0} B_{0}}{(B \rho)}+0=0+\mathcal{W}_{f}=0+\Delta \varepsilon_{f} .
$$

To the very left and very right sides of this equation normalization of the emittances is applied and results into

$$
\Delta \varepsilon_{n f}=\frac{A_{0} B_{0}}{(B \rho)} \beta \gamma=\frac{e A_{0} B_{0}}{m_{0} c},
$$

where $m_{0}$ is the electron rest mass and $e$ is its charge. The authors of [18] used the definition $\mathcal{L}:=\left(e B_{0} A_{0}\right) /\left(2 m_{0} \beta \gamma c\right)$ and hence one obtains for the final difference of eigenemittances

$$
\Delta \varepsilon_{n f}=2 \beta \gamma \mathcal{L}
$$

being exactly the result stated in [18] and fully equivalent to Eq. (29). It is stressed that Eq. (35) has been obtained from Eqs. (21) and (22) without requiring a round beam, while Eq. (29) has been derived by explicitly requiring a round beam [4].

\section{G. Emittance transfer experiment at GSI}

At GSI transverse eigenemittances have been tailored by passing a nitrogen beam through a short solenoid [20]. A stripping foil has been placed at the solenoid center. Doing so, the ions passed the entrance and exit fringe region with different charge state and the imposed vorticities did not compensate each other to zero. Since the exit charge state $(7+)$ has been larger than the entrance charge state $(3+)$, the beam vorticity was effectively changed as through a stand-alone exit fringe field passed with the charge state of 4+. Accordingly, the two eigenemittances have been changed as well. Decoupling of the planes has been performed shortly behind the solenoid by a skewed quadrupole triplet. The parameters of the experiment are listed in Table III.

Calculation of the final difference of eigenemittances compares the parameters in front of the effective standalone exit fringe field, i.e., at the stripping foil right after stripping $\left(B_{s}=B_{f}=0.9 \mathrm{~T}\right)$ to those after the solenoid $\left(B_{s}=0\right)$. Beginning with Eq. (21) gives

$$
\frac{A_{f} B_{f}}{(B \rho)_{\mathrm{eff}}}+0=0+\mathcal{W}_{7+}
$$

i.e., $\mathcal{W}_{7+}=\left(A_{f} B_{f}\right) /(B \rho)_{\text {eff }}$. Using this result and applying Eq. (22) leads to

TABLE III. Beam parameters of the emittance transfer experiment EMTEX [20] in front of and behind the solenoid.

\begin{tabular}{lc}
\hline \hline Parameter & Value \\
\hline Kinetic energy & $11.45 \mathrm{MeV} / \mathrm{u}$ \\
Mass number & 14 \\
$\varepsilon_{x, 3+}$ & $1.040 \mathrm{~mm} \mathrm{mrad}$ \\
$\varepsilon_{y, 3+}$ & $0.825 \mathrm{~mm} \mathrm{mrad}$ \\
$A_{f}$ & $4.166 \mathrm{~mm}^{2}$ \\
$q_{\text {in } / \text { out }}$ & $3+/ 7+$ \\
$q_{\text {eff }}$ & $4+$ \\
$(B \rho)_{3+/ 7+/ 4+}$ & $2.278 / 0.976 / 1.710 \mathrm{Tm}$ \\
$B_{f}$ & $0.9 \mathrm{~T}$ \\
\hline \hline
\end{tabular}




$$
\begin{aligned}
\left(\Delta \varepsilon_{3+}\right)^{2}-0 & =\left(\Delta \varepsilon_{7+}\right)^{2}-\mathcal{W}_{7+}^{2} \\
& =\left(\Delta \varepsilon_{7+}\right)^{2}-\left[\frac{A_{f} B_{f}}{(B \rho)_{\mathrm{eff}}}\right]^{2}
\end{aligned}
$$

and to the final difference of eigenemittances

$$
\Delta \varepsilon_{7+}=\sqrt{\left(\Delta \varepsilon_{3+}\right)^{2}+\left[\frac{A_{f} B_{f}}{(B \rho)_{\mathrm{eff}}}\right]^{2}} .
$$

The same result is obtained by applying Eq. (26). Plugging in the values of Table III together with $\Delta \varepsilon_{3+}=$ $(1.04-0.825) \mathrm{mm}$ mrad delivers

$$
\left|\Delta \varepsilon_{7+}\right|=2.203 \mathrm{~mm} \mathrm{mrad},
$$

being in very good agreement with the measured value of 2.0(4) $\mathrm{mm}$ mrad.

\section{EIGENEMITTANCES AND ROTATIONS}

This section shows that any beam can be regarded as being equivalent to two objects with different areas performing rigid rotations with angular velocities $\pm \omega$ around the beam axis (under the assumptions stated in Sec. IV). To this end we initially refer to the original work of R. Brinkmann [2]. Therein a beam extracted from a cathode being immersed into a solenoidal field is considered. Its phase space coordinates are those of an object rotating with $\omega$, i.e.,

$$
\left[\begin{array}{l}
x_{0} \\
x_{0}^{\prime} \\
y_{0} \\
y_{0}^{\prime}
\end{array}\right]=\left[\begin{array}{c}
x_{0} \\
-\omega y_{0} \\
y_{0} \\
\omega x_{0}
\end{array}\right]
$$

This beam can be transformed into a flat beam inhabiting just horizontal phase space coordinates differing from zero. The according transformation $\mathcal{P}_{x}(\omega)$ is from a beam line segment $M(I, \pi / 2)$, being an identity in the horizontal phase space and providing $\pi / 2$ of phase advance in the vertical space. The segment $M(I, \pi / 2)$ is rotated by $\pi / 4$ around the positive beam axis delivering [2]

$$
\mathcal{P}_{x}(\omega):=R\left(\frac{\pi}{4}\right)\left[\begin{array}{cccc}
1 & 0 & 0 & 0 \\
0 & 1 & 0 & 0 \\
0 & 0 & 0 & 1 / \omega \\
0 & 0 & -\omega & 0
\end{array}\right] R\left(-\frac{\pi}{4}\right)
$$

or

$$
\mathcal{P}_{x}(\omega)=\frac{1}{2}\left[\begin{array}{cccc}
1 & -1 / \omega & 1 & 1 / \omega \\
\omega & 1 & -\omega & 1 \\
1 & 1 / \omega & 1 & -1 / \omega \\
-\omega & 1 & \omega & 1
\end{array}\right] .
$$

Accordingly,

$$
\mathcal{P}_{x}(\omega)\left[\begin{array}{c}
x \\
-\omega y \\
y \\
\omega x
\end{array}\right]=\frac{1}{2}\left[\begin{array}{c}
2 x+2 y \\
2 \omega x-2 \omega y \\
0 \\
0
\end{array}\right]:=\left[\begin{array}{l}
u \\
u^{\prime} \\
0 \\
0
\end{array}\right],
$$

being a flat beam with just horizontal dimensions. These projectors were the base for the round-to-flat transformation suggested and demonstrated by [2] and [16]. They have the properties

$$
\mathcal{P}_{x}^{-1}(\omega)=\mathcal{P}_{x}(-\omega)
$$

$$
\mathcal{P}_{x}^{2}(\omega)=\left[\begin{array}{cccc}
0 & 0 & -1 & 0 \\
0 & 0 & 0 & -1 \\
-1 & 0 & 0 & 0 \\
0 & -1 & 0 & 0
\end{array}\right]
$$

i.e., reflection at $x+y=0$ and $x^{\prime}+y^{\prime}=0$ and hence

$$
\mathcal{P}_{x}^{4}(\omega)=I .
$$

In the following we use the consideration that if $\mathcal{P}_{x}(\omega)$ projects a rotating object into an oscillation in one single transverse plane, application of $\mathcal{P}_{x}^{-1}(\omega)=\mathcal{P}_{x}(-\omega)$ should be the inverted process, that means projection of two transverse oscillations onto two rotating objects.

Any arbitrary distribution may be regarded as a combination of oscillations in the two transverse planes. Application of $\mathcal{P}_{x}^{-1}(\omega)$ to arbitrary particle coordinates gives

$\mathcal{P}_{x}(-\omega)\left[\begin{array}{c}x \\ x^{\prime} \\ y \\ y^{\prime}\end{array}\right]=\frac{1}{2}\left[\left[\begin{array}{c}x+x^{\prime} / \omega \\ -\omega x+x^{\prime} \\ x-x^{\prime} / \omega \\ \omega x+x^{\prime}\end{array}\right]+\left[\begin{array}{c}y-y^{\prime} / \omega \\ \omega y+y^{\prime} \\ y+y^{\prime} / \omega \\ -\omega y+y^{\prime}\end{array}\right]\right]$.

By defining

$$
\begin{aligned}
& a:=x+\frac{x^{\prime}}{\omega}, \quad b:=x-\frac{x^{\prime}}{\omega}, \\
& c:=y-\frac{y^{\prime}}{\omega}, \quad d:=y+\frac{y^{\prime}}{\omega},
\end{aligned}
$$

the previous expression can be restated as 


$$
\mathcal{P}_{x}(-\omega)\left[\begin{array}{c}
x \\
x^{\prime} \\
y \\
y^{\prime}
\end{array}\right]=\frac{1}{2}\left[\begin{array}{c}
a \\
-\omega b \\
b \\
\omega a
\end{array}\right]+\frac{1}{2}\left[\begin{array}{c}
c \\
\omega d \\
d \\
-\omega c
\end{array}\right] \text {, }
$$

describing a superposition of an object $(a b)$ rotating with $\omega$ and an object $(c d)$ rotating with $-\omega$.

Calculation of the eigenemittances corresponding to these two objects is straightforward. As these rotating objects have $\epsilon_{4 d}:=\epsilon_{1} \epsilon_{2}=0$ one eigenemittance is equal to zero and the positive difference of eigenemittances is equal to the other eigenemittance. Applying the findings from Sec. V B, the nonzero eigenemittances of the two objects are

$$
\epsilon_{a b}=2|\omega| A_{a b} \quad \text { and } \quad \epsilon_{c d}=2|\omega| A_{c d},
$$

with $A_{a b}$ and $A_{c d}$ being the rms-areas of these objects. It is left to determine $A_{a b}$ and $A_{c d}$ using the definitions of $a, b$, $c, d$ and Eq. (10). Doing so for $A_{a b}$ one obtains

$$
\begin{aligned}
16 A_{a b}^{2} & =\left\langle a^{2}\right\rangle\left\langle b^{2}\right\rangle-\langle a b\rangle^{2} \\
& =\frac{4}{\omega^{2}}\left\langle x^{2}\right\rangle\left\langle x^{\prime 2}\right\rangle-\frac{4}{\omega^{2}}\left\langle x x^{\prime}\right\rangle \\
& =\frac{4}{\omega^{2}} \epsilon_{x}^{2} .
\end{aligned}
$$

Accordingly, the areas of the objects are simply linked to the beam rms-areas through

$$
A_{a b}=\frac{\epsilon_{x}}{2|\omega|} \quad \text { and } \quad A_{c d}=\frac{\epsilon_{y}}{2|\omega|} .
$$

Comparison of Eqs. (49) and (51) reveals that the eigenemittances of the two objects are equal to the transverse beam rms-emittances

$$
\epsilon_{a b}=\epsilon_{x}, \quad \text { and } \quad \epsilon_{c d}=\epsilon_{y} .
$$

This result has been obtained very rapidly by assuming validity of Eq. (22). It can be confirmed by going through the arduous deviation of plugging the definitions of $a, b, c$, $d$ into Eqs. (2), (3), (5).

\section{CONCLUSIONS AND OUTLOOK}

The quantity $\mathcal{W}$ has been introduced as the beam phase integral or the integrated beam vorticity. For cases of practical interest it was shown that change of difference in transverse eigenemittances is given by the change of the beam phase integral. This is a generalization of previous findings that assigned this difference to angular momentum for the special case of cylindrical symmetric beams, for which the beam phase integral and angular momentum merge. However, for nonsymmetric beams the two quantities are different and the eigenemittance difference is given by the beam phase integral instead.

The new relation drastically simplifies calculations of eigenemittances as well as quantitative modeling of experiments that tailor beam eigenemittances. Additionally, it allows for gaining an improved physical picture of the nature of eigenemittances. Beams are equivalent to superposition of two rotating objects rotating with $\pm \omega$ inhabiting rms-areas being equal to the beam transverse rms-emittances divided by $2|\omega|$.

As final remark we notice the occurrence and relevance of phase integrals in the frame of beam emittance variation through longitudinal magnetic fields. Recently the variation of single particle angular momentum in such environments has been revisited with the perspective of quantization of this angular momentum [35,36]. Future works may address whether this concept could be extended to beam emittances.

\section{APPENDIX: ILLUSTRATION OF DIFFERENCE BETWEEN BEAM ANGULAR MOMENTUM AND PHASE INTEGRAL}

This paragraph shall illustrate the difference between angular momentum

$$
L=\left\langle x y^{\prime}\right\rangle-\left\langle y x^{\prime}\right\rangle
$$

and beam phase integral

$$
\mathcal{W}=\frac{1}{A}\left[\left\langle y^{2}\right\rangle\left\langle x y^{\prime}\right\rangle-\left\langle x^{2}\right\rangle\left\langle y x^{\prime}\right\rangle+\langle x y\rangle\left(\left\langle x x^{\prime}\right\rangle-\left\langle y y^{\prime}\right\rangle\right)\right],
$$

with $A=\sqrt{\left\langle x^{2}\right\rangle\left\langle y^{2}\right\rangle-\langle x y\rangle^{2}}$ together with their role in the context of eigenemittances. The first example treats an ellipse performing two different types of rotation and the second is on a thin wire moving along a rectangular orbit.

\section{Rotating ellipse}

The ellipse depicted in Fig. 8 starts a rigid rotation around its center and accordingly its second moments $\left\langle x^{2}\right\rangle$, $\left\langle y^{2}\right\rangle$, and $\langle x y\rangle$ vary during rotation. $L_{\text {rig }}, \mathcal{W}_{\text {rig }}$, and the two

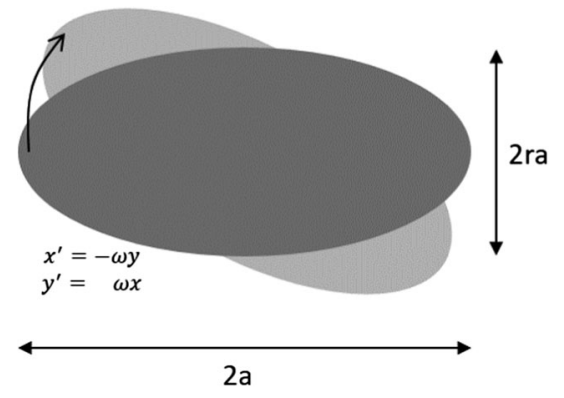

FIG. 8. Ellipse performing rigid and concentric rotation. 


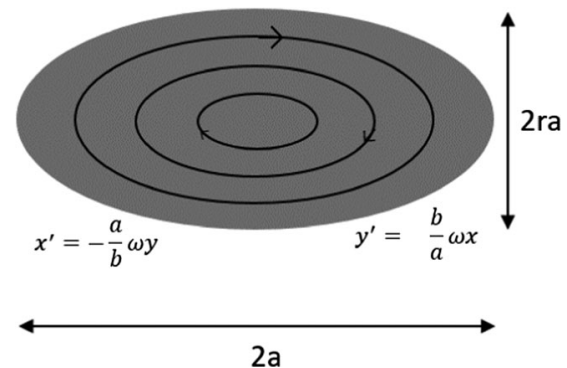

FIG. 9. Ellipse performing intrinsic and concentric rotation.

eigenemittances $\epsilon_{1 / 2, \text { rig }}$ remain constant. Calculation of these four quantities from the second moments delivers

$$
\begin{aligned}
L_{\text {rig }} & =\frac{\omega}{4} a^{2}\left(1+r^{2}\right), \quad \mathcal{W}_{\text {rig }}=\frac{\omega}{2} a^{2} r, \\
\epsilon_{1, \text { rig }} & =\Delta \epsilon=\mathcal{W}_{\text {rig }} \leq L_{\text {rig }}, \quad \epsilon_{2, \text { rig }}=0 .
\end{aligned}
$$

Here the eigenemittances were calculated by applying Eqs. (2), (3), (5).

Instead in the case depicted in Fig. 9 the ellipse performs an intrinsic rotation preserving its second moments $\left\langle x^{2}\right\rangle,\left\langle y^{2}\right\rangle$, and $\langle x y\rangle$. Recalculation of $L, \mathcal{W}$, and $\epsilon_{1 / 2}$ results into

$$
\begin{aligned}
L_{\text {int }} & =\frac{\omega}{2} a^{2} r, \quad \mathcal{W}_{\text {int }}=\frac{\omega}{4} a^{2}\left(1+r^{2}\right), \\
\epsilon_{1, \text { int }} & =\Delta \epsilon=\mathcal{W}_{\text {int }} \geq L_{\text {int }}, \quad \epsilon_{2, \text { int }}=0 .
\end{aligned}
$$

Comparison of rigid and intrinsic rotation reveals that swapping the type of rotation swaps the expressions for $L$ and $\mathcal{W}$. Nonetheless, for both types the difference of eigenemittances $\Delta \varepsilon$ is given by the beam path integral $\mathcal{W}$ and not by the angular momentum $L$. Just for the special case of cylindrical symmetry $r=1$ the two types of rotation merge as do the two quantities, i.e., $L=\mathcal{W}=\omega a^{2} / 2$.

\section{Moving wire}

The second example considers a thin wire starting to move with constant velocity $u^{\prime}$ around a rectangular area as depicted in Fig. 10. Calculation of its acquired angular momentum, beam phase integral or vorticity, and

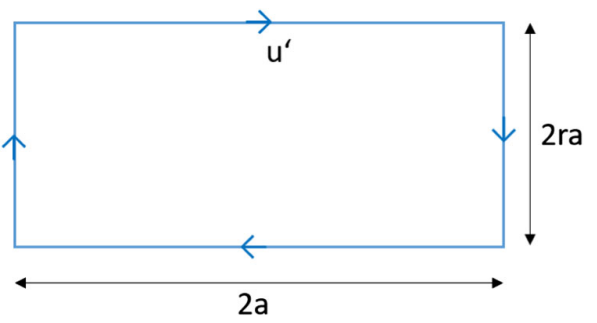

FIG. 10. Thin wire moving around a rectangular area. eigenemittances is straight forward. It is lengthy for the eigenemittances using Eqs. (2), (3), (5) and it delivers

$$
\begin{aligned}
L & =a u^{\prime} \cdot l(r) \\
\mathcal{W} & =a u^{\prime} \cdot w(r) \\
\varepsilon_{1 / 2} & =a u^{\prime} \cdot e_{1 / 2}(r),
\end{aligned}
$$

with

$$
\begin{aligned}
l(r) & =\frac{2 r}{1+r} \\
w(r) & =\frac{(1+r)^{2}}{\sqrt{3 r^{2}+10 r+3}}
\end{aligned}
$$

and

$$
e_{1}(r)-e_{2}(r)=\sqrt{\frac{r^{2}+r+1}{3}}
$$

from Eq. (6).

As for the ellipse these quantities depend on the geometric aspect ratio $r$ and the $r$-dependence of the angular momentum $l(r)$ is different with respect to the one of the beam phase integral and the difference of eigenemittances. While the two latter scale proportional to $r$ for large $r$, the angular momentum converges to $l(r)=2$. At first glance the expressions of $w(r)$ and of the difference of eigenemittances seem different, but they are actually almost identical. Figure 11 plots the angular momentum, the phase integral, and the eigenemittances as functions of the aspect ratio $r$. It reveals that the difference of eigenemittances is very well approximated by the phase integral (the relative difference is less than $1 \%)$. Comparing $w(r)$ to $e_{1 / 2}(r)$, the derivation of $w(r)$ is significantly simpler and faster.

The angular momentum instead is obviously systematically off from the difference of eigenemittances. Just

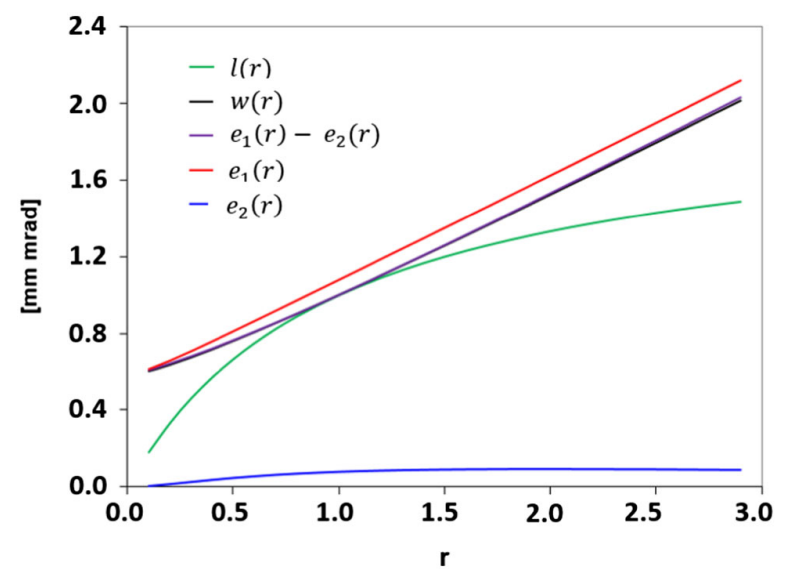

FIG. 11. Angular momentum (green), beam phase integral or vorticity (black), difference of eigenemittances (violet), and the two eigenemittances (red and blue) as functions of the geometric aspect ratio $r$. 
for the special case of symmetry $r=1$, the phase integral or vorticity is equal to the angular momentum, i.e., $l(1)=w(1)=e_{1}(1)-e_{2}(1)=1$. Hence, another evidence has been provided that change of difference of eigenemittances is generally given by change of vorticity and not by change of angular momentum.

It is also illustrative to pick the opposite case of $r \rightarrow 0$. The rigidly rotating ellipse inhabits angular momentum but no vorticity, while the intrinsically rotating ellipse has vorticity but no angular momentum.

[1] A. J. Dragt, General moment invariants for linear Hamiltonian systems, Phys. Rev. A 45, 2572 (1992).

[2] R. Brinkmann, Y. Derbenev, and K. Flöttmann, A low emittance, flat-beam electron source for linear colliders, Report No. DESY TESLA-99-09, 1999.

[3] A. Burov, S. Nagaitsev, and Y. Derbenev, Circular modes, beam adapters, and their applications in beam optics, Phys. Rev. E 66, 016503 (2002).

[4] K.-J. Kim, Round-to-flat transformation of angularmomentum-dominated beams, Phys. Rev. Accel. Beams 6, 104002 (2003).

[5] P. Bertrand, J. P. Biarrotte, and D. Uriot, Flat Beams and application to the mass separation of radioactive beams, in Proceedings of the 10th European Particle Accelerator Conference, Edinburgh, Scotland, edited by J. Poole and C. Petit-Jean-Genaz (Institute of Physics, Edinburgh, Scotland, 2006).

[6] P. Emma, Z. Huang, K.-J. Kim, and P. Piot, Transverse-tolongitudinal emittance exchange to improve performance of high-gain free-electron lasers, Phys. Rev. Accel. Beams 9, 100702 (2006).

[7] L. Groening, Concept for controlled transverse emittance transfer within a linac ion beam, Phys. Rev. Accel. Beams 14, 064201 (2011).

[8] D. Xiang and A. Chao, Emittance and phase space exchange for advanced beam manipulation and diagnostics, Phys. Rev. Accel. Beams 14, 114001 (2011).

[9] B. E. Carlsten, K. A. Bishofberger, L. D. Duffy, S. J. Russell, R. D. Ryne, N. A. Yampolsky, and A. J. Dragt, Arbitrary emittance partitioning between any two dimensions for electron beams, Phys. Rev. Accel. Beams 14, 050706 (2011).

[10] B. E. Carlsten, K. A. Bishofberger, S. J. Russell, and N. A. Yampolsky, Using an emittance exchanger as a bunch compressor, Phys. Rev. Accel. Beams 14, 084403 (2011).

[11] L. D. Duffy, K. A. Bishofberger, B. E. Carlsten, A. Dragt, Q. R. Marksteiner, S. J. Russell, R. D. Ryne, and N. A. Yampolsky, Exploring minimal scenarios to produce transversely bright electron beams using the eigenemittance concept, Nucl. Instrum. Methods Phys. Res., Sect. A 654, 52 (2011).

[12] R. C. McCrady, K. Bishofberger, and B. E. Carlsten, A tapered-foil emittance-exchange experiment at LANSCE, Proceedings of the 2nd International Particle Accelerator Conference, New Orleans, U.S.A., edited by J. Corbett (IEEE, Piscataway, NJ, 2012).
[13] C. Xiao, L. Groening, O. Kester, H. Leibrock, M. Maier, and C. Mühle, Single-knob beam line for transverse emittance partitioning, Phys. Rev. Accel. Beams 16, 044201 (2013).

[14] C. Xiao, L. Groening, and O. K. Kester, Collimation and decoupling of ECR source beams for brilliance optimization, Nucl. Instrum. Methods Phys. Res., Sect. A 738, 167 (2014).

[15] L. Groening, Decoupling of beams previously coupled by effective stand-alone solenoid fringe fields, arXiv:1403.6962.

[16] D. Edwards, H. Edwards, N. Holtkamp, S. Nagaitsev, J. Santucci, R. Brinkmann, K. Desler, K. Flöttmann, I. Bohnet, and M. Ferrario, The flat beam experiment at the FNAL photoinjector, in Proceedings of the $X X$ Linear Accelerator Conference, Monterey, CA, edited by A. Chao, e000842 (2000), https://accelconf.web.cern.ch/100/papers/ MOB13.pdf.

[17] R. Brinkmann, Y. Derbenev, and K. Flöttmann, A low emittance, flat-beam electron source for linear colliders, Phys. Rev. Accel. Beams 4, 053501 (2001).

[18] P. Piot, Y.-E Sun, and K.-J. Kim, Photoinjector generation of a flat electron beam with transverse emittance ratio of 100, Phys. Rev. Accel. Beams 9, 031001 (2006).

[19] Y.-E. Sun, P. Piot, A. Johnson, A. H. Lumpkin, T. J. Maxwell, J. Ruan, and R. Thurman-Keup, Tunable Subpicosecond Electron-Bunch-Train Generation Using a Transverse-To-Longitudinal Phase-Space Exchange Technique, Phys. Rev. Lett. 105, 234801 (2010).

[20] L. Groening, M. Maier, C. Xiao, L. Dahl, P. Gerhard, O. K. Kester, S. Mickat, H. Vormann, M. Vossberg, and M. Chung, Experimental Proof of Adjustable Single-Knob Ion Beam Emittance Partitioning, Phys. Rev. Lett. 113, 264802 (2014).

[21] S. Appel, L. Groening, Y. El Hayek, M. Maier, and C. Xiao, Injection optimization through injection of flat beams, Nucl. Instrum. Methods Phys. Res., Sect. A 866, 36 (2017).

[22] M. Chung, H. Qin, R. Davidson, L. Groening, and C. Xiao, Generalized Kapchinskij-Vladimirskij distribution and beam matrix for phase-space manipulations of highintensity beams, Phys. Rev. Lett. 117, 224801 (2016).

[23] E. Prat and M. Aiba, Four-dimensional transverse beam matrix measurement using the multiple-quadrupole scan technique, Phys. Rev. Accel. Beams 17, 052801 (2014).

[24] J. Ögren, R. Ruber, V. Ziemann, and W. Farabolini, Measuring the full transverse beam matrix using a single octupole, Phys. Rev. Accel. Beams 18, 072801 (2015).

[25] C. Xiao, L. Groening, P. Gerhard, M. Maier, S. Mickat, and H. Vormann, Measurement of the transverse fourdimensional beam rms-emittance of an intense uranium beam at 11.4 MeV/u, Nucl. Instrum. Methods Phys. Res., Sect. A 820, 14 (2016).

[26] C. Xiao, M. Maier, X. N. Du, P. Gerhard, L. Groening, S. Mickat, and H. Vormann, Rotating system for fourdimensional transverse rms-emittance measurements, Phys. Rev. Accel. Beams 19, 072802 (2016).

[27] B. Cathey, S. Cousineau, A. Aleksandrov, and A. Zhukov, First Six Dimensional Phase Space Measurement of an Accelerator Beam, Phys. Rev. Lett. 121, 064804 (2018). 
[28] C. Xiao, X. N. Du, L. Groening, and M. Maier, Refining the evaluation of eigen emittances measured by the dedicated four-dimensional emittance scanner ROSE, Nucl. Instrum. Methods Phys. Res., Sect. A 964, 163828 (2020).

[29] Using

$$
\varepsilon_{1 / 2}=\sqrt{\varepsilon_{4 d}+\frac{\Delta \varepsilon}{4}} \pm \frac{\Delta \varepsilon}{2}
$$

with $\Delta \varepsilon:=\varepsilon_{1}-\varepsilon_{2} \geq 0$ and $\varepsilon_{1}^{2}+\varepsilon_{2}^{2}=-\frac{\operatorname{tr}\left([C J]^{2}\right)}{2}$ from Eq. (5) one obtains $\Delta \varepsilon=\sqrt{-2 \varepsilon_{4 d}-\frac{\operatorname{tr}\left([C J]^{2}\right)}{2}}$ and finally the alternative expressions for $\varepsilon_{1 / 2}$.

[30] K. Floettmann, Some basic features of the beam emittance, Phys. Rev. Accel. Beams 6, 034202 (2003).

[31] H. Busch, Berechnung der Bahn von Kathodenstrahlen in axial symmetrischen electromagnetischen Felde, Ann. Phys. (Berlin) 386, 974 (1926).
[32] L. Groening, C. Xiao, and M. Chung, Extension of Busch's theorem to particle beams, Phys. Rev. Accel. Beams 21, 014201 (2018).

[33] It can be shown that $\mathcal{W}$ from Eq. (11) is invariant under rotation around the beam axis.

[34] It is restressed that these statements refer to the laboratory coordinates $\left(x, y, x^{\prime}, y^{\prime}\right)$ being relevant for applications. In conjugate coordinates the eigenemittances are preserved also along solenoid main and fringe fields. This preservation is the base of the extended Busch theorem [32].

[35] K. Floettmann, Quantum mechanical formulation of the Busch theorem, Phys. Rev. A 102, 043517 (2020).

[36] D. V. Karlovets, Vortex particles in axially symmetric fields and applications of the quantum Busch theorem, New J. Phys. 23, 033048 (2021). 